\title{
Understanding the Use of loT Systems as Architecture Design Stimuli
}

\author{
Tao Shen*, Yukari Nagai \\ Knowledge Science, Japan Advanced Institute of Science and Technology, Nomi City, Japan \\ Email address: \\ shentao@jaist.ac.jp (T. Shen) \\ ${ }^{*}$ Corresponding author \\ To cite this article: \\ Tao Shen, Yukari Nagai. Understanding the Use of IoT Systems as Architecture Design Stimuli. International Journal of Architecture, Arts \\ and Applications. Special Issue: Innovation in Architecture Design. Vol. 5, No. 2, 2019, pp. 42-49. doi: 10.11648/j.ijaaa.20190502.12
}

Received: June 24, 2019; Accepted: July 27, 2019; Published: September 2, 2019

\begin{abstract}
We are entering a new area of information science that we calling the Internet of Things (IoT). It connects machine with machine, machine with infrastructure and machine with environment, the Internet of everything. More generally, we see IoT as massive amounts of connected concepts that encompass every aspect of our lives. Meanwhile, architects often explore novel design ideas of their knowledge and skills for innovation, even though such ideas rely on their own experience, expertise or intuition, so that it brings the negative effects on creative architecture design. Numerous studies have investigated that concept-synthesizing processes is a key to creative design. However, there is little work specifically on understanding the use of IoT systems as architecture design stimuli. In this paper, we present a model of using IoT systems as design stimuli for architecture concept generation, in this model we abstract IoT systems into 'input part', 'process part' and 'output part'. Through a controlled experiment and extended protocol analysis, this research showed that IoT systems stimulate creative architecture design both in design process and design result, in addition, participants often choose the 'input part' as design stimuli while 'input part' and 'output part' both have the promoter action to creativity. Moreover, 'process part' prefers to enhance the extension of idea space in concept generation process.
\end{abstract}

Keywords: Cognitive Process, Concept Generation, Creative Stimulation, Architecture Design, IoT System

\section{Introduction}

Today, the electrification of the world is around us. Almost any manufactured good now includes an embedded processor, such as a microcontroller. Being along with user interfaces, that can add the functionality of programmability and deterministic 'command and control' [1]. In general, we can see the IoT as a vast number of connected 'things' which encompass every aspect of our lives, these 'things' can be smart or non-smart objects, also can be biological or natural entities such as humans and animals. No matter 'things' are devices or natural entities, we can abstract them as massive amounts of concepts, therefore, IoT systems also mean the systems of connected massive amounts of concepts, it is the key to understand using IoT Systems as design stimuli for concept generation.

In this paper, we use extended protocol analysis method to examine how designers use IoT systems as design stimuli during concept generation.

\section{Theoretical Background}

\subsection{Design Stimuli}

Many studies have been conducted to analyze the essence of the design process, and many significant experiments have identified its creative features within a problem-solving framework [2]. Chiu and Shu proposed a methodology that use language as stimuli to enhance the concept generation process in the design process. They presented the results of experiments where verbal protocols were used to provoke information on how designers used semantic stimuli presented as words related to the problem during concept generation and they found people often used stimuli in noun form but more new ideas come out when people use stimuli as verbs and noun modifiers [3]. However, Benami and Jin [4] developed a cognitive model of creative conceptual 
design to capture the relationship between the properties that stimulate cognitive processes. They classified creative stimulation into function stimulation, form stimulation, behavior stimulation and knowledge entity stimulation.

1. Function Stimulation: A design task for fulfilling users' needs. For example, in designing a house a designer may consider function 'big enough for a family living' or 'heat preservation' to fulfill living requirements.

2. Form Stimulation: The shape and structure of a component of the design artifact. Designers generate mental images of products in the early stage of design.

3. Behavior Stimulation: The means where something works. Behaviors can be divided into a designed response or an unintentional response. Many designs usually contain several types of behavior. For instance, in designing washing machine, there are many behaviors may occur, such as the number of cycles that the machine can control, the water temperature, and the signals that are sent to user.

4. Knowledge Entity Stimulation: A design entity comprise functions, forms, and behaviors, and they can be classified as preinventive entity at the point of inception. The knowledge entity is evolved from a preinventive entity. For example, bicycle is a knowledge entity stimulation and a very common entity, therefore, it's easy to make analogies to the functions, forms, and behaviors in the bicycle, which would stimulate new design ideas.

It is difficult to use language to achieve all these stimulation's function, especially when we use language stimuli at the word level. However, IoT systems are able to provide more stimulation patterns for designers. For example, if the output is a 'fan', it can stimulate designers the function 'keep cool' or 'wind source', when the fan works, it can stimulate designers the form 'circle' and behaviors such as 'create flow within a fluid'. Therefore, we present a model of using IoT systems as design stimuli for concept generation.

\subsection{Concept Synthesis}

In the concept generation process, we adopt the process of synthesizing two base concepts as a framework to get the concept generation process in design process. The reason we adopt this process is that it is the simplest and most essential process for generating a new concept from existing comcepts [5] [6]. Furthermore, Nagai and Taura [7] presented three reasons why adopting the process of synthesizing. The first reason is related to the empirical aspect. In an actual field, the concept synthesizing process can be found. For example, the invention of the art knife stemmed from the synthesis of two base concepts, a kind of chocolate which segments can be broken off and the sharp edges of broken glass [8]. The second reason is related to the framing aspect. Property mapping, concept blending, and concept integrating are typical important concept generation processes, the concept synthesizing process involves these processes. The third reason is related to the experimental aspect. In the field of linguistic studies, numerous studies have been accumulated from the study of noun-noun phrases [9, 10]. As considering the base concepts as a compound phrase composed of two nouns, people can compare the design process with the linguistic interpretation process. For example, through the hybrid linking process, a knife-shaped fork is considered as a knife and fork set used together while eating; through the property mapping process, that is considered as one-half as a knife and the other half as a fork.

As a result, concept-synthesizing processes have been found to be a key to creative design [11]. In IoT systems, large amounts of concepts are connected with each other, this property make IoT systems play important roles in concept generation.

\subsection{IoT Systems as Design Stimuli}

As design stimuli, we classify IoT systems into 'input part', 'process part' and 'output part', 'process part' connects 'input part' with 'output part' and provide logic for connection. Figure 1 shows the model we present using IoT systems as design stimuli for concept generation. 'input part' and 'output part' give designers stimulus A and B which can be function stimulation, form stimulation, behavior stimulation or knowledge entity stimulation. 'process part' also participate in the concept synthesizing process, which make stimulus $\mathrm{A}$ and $\mathrm{B}$ change under different conditions and give a logic relationship between stimulus $\mathrm{A}$ and $\mathrm{B}$. That is the most important and different point comparing with other methods which seek design stimuli in concept generation. Our main research questions were: What is the effort of presenting IoT systems as design stimuli on concept generation? And will a genealogical linkage occur between 'process part' and concept generation process?

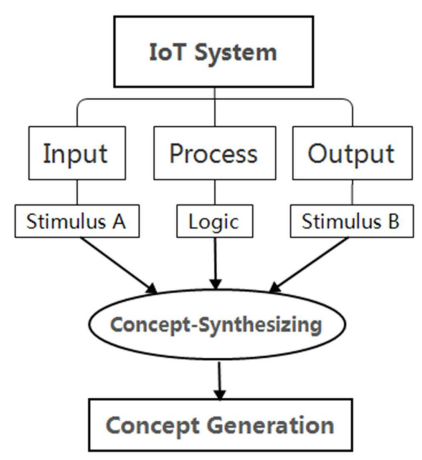

Figure 1. The model of using IoT systems as design stimuli.

\section{Experimental Methods}

In this research, we developed the extended protocol method created by Taura and Nagai [12].

\subsection{Participants}

In this experiment, participants consisted of twenty master students in knowledge science, all the twenty students are not experienced designers. Therefore, we evaluated their 
creativity based on the basis of the design concept.

\subsection{Analysis}

In order to elucidate the stimulatory effect of using IoT systems in concept generation process, we analyzed not only the design ideas generated by participants but also their performance in the design process, as we can get important keys according to Lawson [13] from the design thinking process.

\subsubsection{Analysis of Design Process}

In this experiment, we use protocol analysis and semistructured interviews. Participants are asked to 'think aloud' during the design process therefore we can gather their utterances as protocol data for designing [14]. After the design process, participants are asked to explain the reasons why they made such design activities while observing the videos of their performance of the design, the reason for that is participants do not always show their reasons behind their thinking when they are asked to say aloud during design process.

\subsubsection{Analysis of Design Results}

To analyze the design results of participants, we used the method developed by Finke et al [15]. By using this method, we evaluated participants' design results based on practicality and originality with a five points scale.

\subsection{Design Task}

Twenty participants were divided into group A and group $\mathrm{B}$ at random. Both groups were asked to design a new architecture idea starting from the IoT system modules we provided to them, as building is very familiar to participants and they can easily propose their ideas about new building. So that to make participants easily use IoT systems, we adopt 'mCookie Series' developed by Microduino Company, which are modular, stackable, building blocks compatible electronics platform such as Arduino, Mixly and Scratch for programming.

The different point between group A and group B is that the 'process part' is only provided to group B, they can use Mixly (a free and simple programming language) to create their own 'process part'.

Table 1. The 'Input parts' and 'Output parts' used by group A and group B in this experiment.

\begin{tabular}{lll}
\hline No & Input & Output \\
\hline 1 & Light sensor & Colorful LED light \\
\hline
\end{tabular}

\begin{tabular}{lll}
\hline No & Input & Output \\
\hline 2 & Sound sensor & Speaker \\
3 & Motion sensor & Motor \\
4 & Temperature sensor & Temperature controller \\
5 & Real-Time Clock & Timer \\
\hline
\end{tabular}

The reason we adopted these IoT modules is that these modules are quite common and representative in IoT systems. In addition, each group has the same original concept, corresponding to each group, the concepts are light, sound, motion, temperature and time.

\subsection{Experiment Process}

\subsubsection{Preparation (20 or 30 Minutes)}

Participants are instructed to 'think-aloud' and verbalize all thoughts and reasoning by given a series of simple tasks, meanwhile they are trained to use 'mCookie Series' to create IoT systems. Group B also learnt to use Scratch in the extra 10 minutes.

\subsubsection{Design (20 Minutes)}

In this session, participants are asked to 'think-aloud' during performing the design task. Meanwhile we use video camera to record their utterances and behavior. Therefore, we can get the protocol data and design result.

\subsubsection{Interview (30 Minutes)}

In this session, participants are asked to watch the video and explain the reasons for their design behavior. So that we can make clear the reasons behind the concepts they generated.

\subsubsection{Creativity Evaluation}

The design results are evaluated by 8 people including 4 professors majored in architecture design. They evaluated these design results based on practicality and originality on a five-point scale.

\subsection{Process Observations and Design Results}

Twenty participants finally produced 20 new architecture ideas, during the design process, group A can quickly choose the modules they want and give their ideas immediately, most participants in group B develop their ideas with the formation of their logic presented by Mixly. Because the 20 participants are not experienced in architecture design, we summarized these ideas as design concept. Table 2 shows these concepts and the number of stimulus modules associated with each concept.

Table 2. The 20 concepts and the number of stimulus modules associated with each concept.

\begin{tabular}{lllll}
\hline Group & No. & Architecture Concept & Number of Input modules & Number of Output modules \\
\hline A & 1 & A restaurant with changeable light & 3 & 1 \\
A & 2 & A cinema with visual wall & 3 & 4 \\
A & 3 & A collapsible house & 4 & 4 \\
A & 4 & A kindergarten with much monitoring device & 4 & 4 \\
A & 5 & An automatic control live house & 5 & 3 \\
A & 6 & A science museum like spaceship & 5 & 5 \\
\hline
\end{tabular}




\begin{tabular}{lllll}
\hline Group & No. & Architecture Concept & Number of Input modules & Number of Output modules \\
\hline A & 7 & A villa with changeable building skin & 2 & 2 \\
A & 8 & A comfortable kindergarten & 2 & 3 \\
A & 9 & A kindergarten like Disneyland & 3 & 3 \\
A & 10 & A gym like a forest & 3 & 2 \\
B & 11 & An unmanned hotel & 3 & 3 \\
B & 12 & A house with eyes & 3 & 2 \\
B & 13 & A hall with virtual stages & 3 & 4 \\
B & 14 & A subway station with flow guidance system & 4 & 3 \\
B & 15 & A breathing library & 5 & 4 \\
B & 16 & A gym with smart control system & 5 & 5 \\
B & 17 & A hotel which can identify guest & 5 & 1 \\
B & 18 & A re-generator Skyscraper & 5 & 3 \\
B & 19 & A pyramid with entire ecosystem & 5 & 4 \\
B & 20 & A stealthy observation station & 5 & 2 \\
\hline
\end{tabular}

\section{Analysis and Results}

\subsection{Tendency to Use 'Input Part' and 'Output Part'}

In order to investigate the tendency to use 'input part' and 'output part' as design stimuli, we took the paired sample t-test and Table 3 shows the result of this analysis and it is significant. It is understood that although the original concept is same, participants have the tendency to use 'input part' as design stimuli.

Table 3. The result of paired sample t-test on the number of 'input part' and 'output part'.

\begin{tabular}{llll}
\hline & $\boldsymbol{N}$ & Mean & SD \\
\hline Input & 20 & 3.85 & 1.089 \\
Output & 20 & 3.10 & 1.165 \\
Sig. (2-tailed) & 0.021 & & \\
\hline
\end{tabular}

\subsection{Creativity Evaluation}

According to the judging standard, if the practicality rating is less than 3 points, the design idea is not qualified. Therefore, we finally get 18 ideas satisfy the judging standard. Table 4 shows the average rating for the 18 ideas and the total number of stimulus modules associated with each concept.

Table 4. The creativity evaluation of 18 ideas and the total number of stimulus modules associated with each concept.

\begin{tabular}{|c|c|c|c|c|}
\hline Group & No. & Originality & Practicality & Number of modules \\
\hline A & 1 & 2 & 3.25 & 4 \\
\hline A & 2 & 3.625 & 3.125 & 7 \\
\hline A & 3 & 3.75 & 3.125 & 8 \\
\hline A & 4 & 3 & 3.75 & 8 \\
\hline A & 5 & 3.625 & 3.5 & 8 \\
\hline A & 7 & 2.125 & 3.125 & 4 \\
\hline A & 8 & 2.625 & 3.375 & 5 \\
\hline A & 9 & 2.75 & 3.625 & 6 \\
\hline A & 10 & 3.625 & 3.125 & 5 \\
\hline B & 11 & 3.125 & 3.5 & 6 \\
\hline B & 12 & 3 & 3 & 5 \\
\hline B & 13 & 3.75 & 3.5 & 7 \\
\hline B & 14 & 3.625 & 3.25 & 7 \\
\hline B & 15 & 4 & 3.125 & 9 \\
\hline B & 17 & 1.875 & 3 & 6 \\
\hline B & 18 & 3.875 & 3 & 8 \\
\hline B & 19 & 4 & 3.125 & 9 \\
\hline B & 20 & 3.75 & 3 & 7 \\
\hline
\end{tabular}



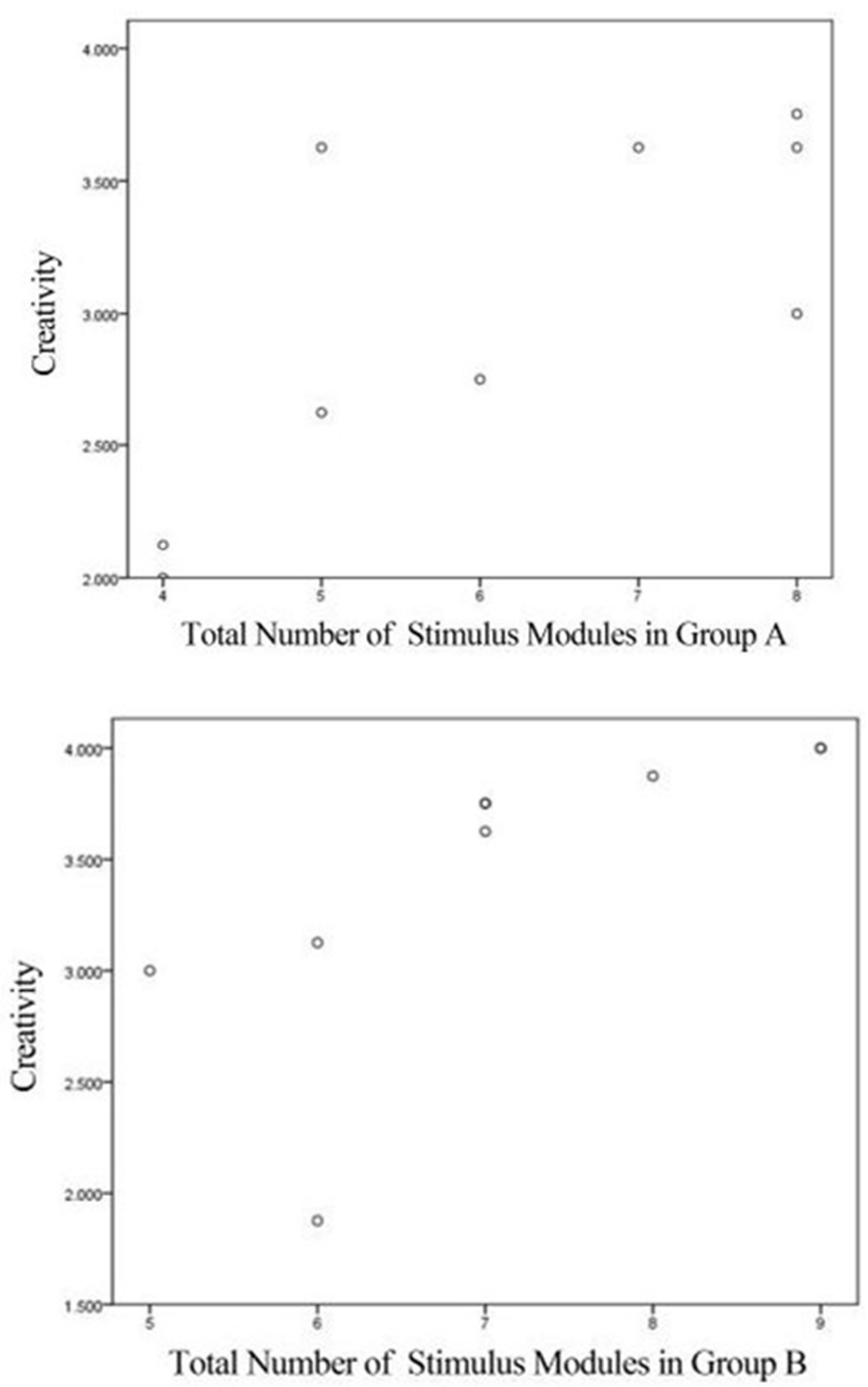

Figure 2. Correlation between creativity and total number of stimulus modules of group A and B.

Table 5 shows the mean and standard deviation of creativity and total number of stimulus modules for each group. Figure 2 shows the scatter charts.

Table 5. The mean and standard deviation of creativity and total number of stimulus modules.

\begin{tabular}{cllrll}
\hline Group A & Creativity & $\begin{array}{l}\text { Number of } \\
\text { modules }\end{array}$ & Group B & Creativity & $\begin{array}{l}\text { Number of } \\
\text { modules }\end{array}$ \\
\hline $\bar{X}$ & 3.014 & 6.110 & $\mathrm{X}$ & 3.444 & 7.11 \\
$\mathrm{SD}$ & 0.680 & 1.691 & $\mathrm{SD}$ & 0.688 & 1.364 \\
\hline
\end{tabular}

The Pearson correlation coefficient of group A is 0.745 , $p=0.021<0.05$, the Pearson correlation coefficient of group B is $0.723, p=0.028<0.05$. Both are significant, it indicates that no matter whether the 'process part' exists, there is a strong correlation between creativity and the number of modules in IoT systems.

Table 6 shows the regression analysis result of total number of stimulus modules to creativity. Both groups are significant, it is understood that 'input part' and 'output part' in IoT systems have the promoter action to creativity. 
Table 6. The regression analysis result of total number of stimulus modules to creativity.

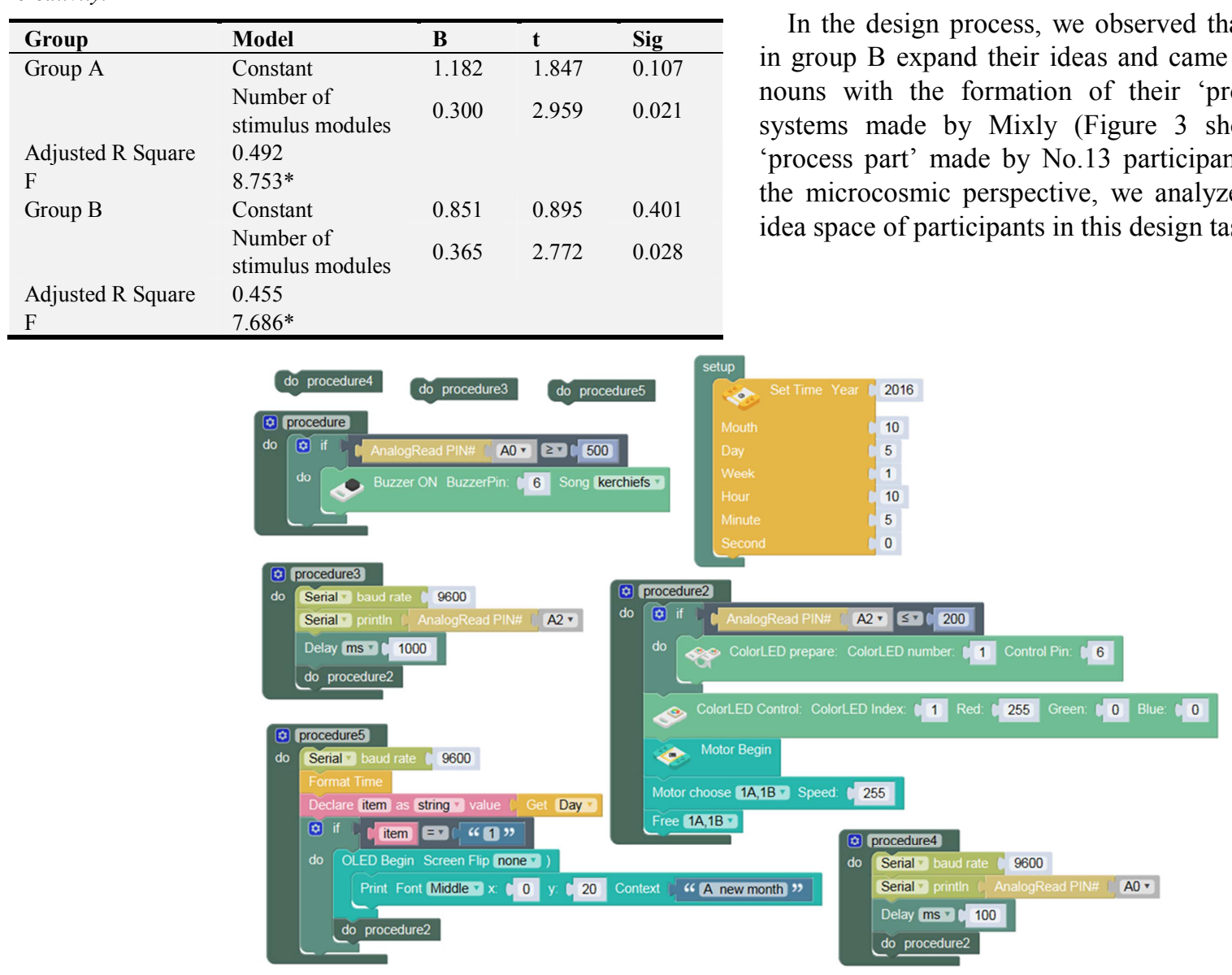

Figure 3. Process part made by No.13 participant.

To identify the extension of idea space, we get new nouns extracted from the utterances recorded in the design process and interview. Next step is measuring conceptual distance, in the previous research [12], the distance between newly uttered noun and basic concepts is measured by counting the number of nodes along the shortest path between the concepts. In this research, we compute the distances between

\section{Extension of Idea Space}

In the design process, we observed that most participants in group B expand their ideas and came up with many new nouns with the formation of their 'process part' in IoT systems made by Mixly (Figure 3 shows an sample of 'process part' made by No.13 participant). Therefore, from the microcosmic perspective, we analyzed the extension of dea space of participants in this design task.

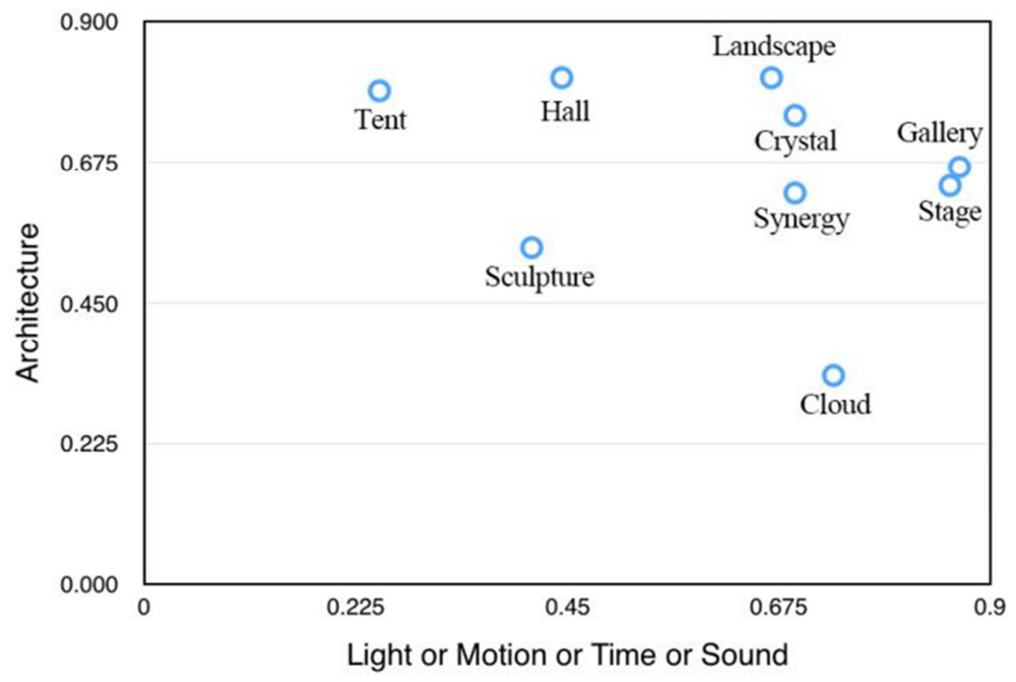

Figure 4. The distance of the new nouns from architecture and associated stimulus modules' concept in No. 13. 
If (is a new noun, then we define the extension of design space is $(\mathrm{N}=$ number of new nouns). We compared the extension of design space of participants in Group A and Group B who used the same number of stimulus modules, Table 7 shows the result.

Table 7. The extension of design space comparison between Group A and Group B.

\begin{tabular}{llll}
\hline Group & No. & Number of modules & Extension of design space \\
\hline A & 8 & 5 & 0.356 \\
A & 10 & 5 & 0.421 \\
B & 12 & 5 & 0.445 \\
A & 9 & 6 & 0.416 \\
B & 11 & 6 & 0.579 \\
B & 17 & 6 & 0.378 \\
A & 2 & 7 & 0.634 \\
B & 13 & 7 & 0.770 \\
B & 14 & 7 & 0.712 \\
B & 20 & 7 & 0.793 \\
A & 3 & 8 & 0.677 \\
A & 4 & 8 & 0.502 \\
A & 5 & 8 & 0.657 \\
B & 18 & 8 & 0.796 \\
\hline
\end{tabular}

Table 8 shows the mean of the extension of idea space between Group A and Group B with the same number of modules. Table 9 shows the result of the paired sample t-test and it is significant. The results indicate that when the number of stimulus modules is same, 'process part' in IoT systems enhance the extension of idea space.

Table 8. The mean of the extension of idea space between Group A and Group B same number of modules.

\begin{tabular}{lll}
\hline $\begin{array}{l}\text { Number of } \\
\text { modules }\end{array}$ & $\begin{array}{l}\text { Mean of the extension of } \\
\text { idea space in Group A }\end{array}$ & $\begin{array}{l}\text { Mean of the extension of } \\
\text { idea space in Group B }\end{array}$ \\
\hline 5 & 0.389 & 0.445 \\
6 & 0.416 & 0.479 \\
7 & 0.634 & 0.758 \\
8 & 0.612 & 0.796 \\
\hline
\end{tabular}

Table 9. The result of paired sample t-test on the extension of idea space between Group A and Group B.

\begin{tabular}{llll}
\hline & N & Mean & SD \\
\hline Group A & 4 & 0.513 & 0.128 \\
Group B & 4 & 0.620 & 0.183 \\
Sig. (2-tailed) & 0.038 & & \\
\hline
\end{tabular}

\section{Discussion}

From the experimental observation, it appears that the participants in Group A came up with most of the new nouns in the beginning of the design process, however, the rate of generation of new nouns reduced significantly with time. This validates the work performed by Howard [18]. In contrast, most participants in Group B constantly came up with new nouns throughout the design process. The process part stimulus was observed to trigger a secondary stimulus that is quite useful. It can also be seen from Table 8 that the participants using the process part stimulus have a higher extension of the idea space with the same number of modules. Thus, we define the effect of the process part stimulus as a 'catalytic stimulus'.

Hatchuel and Weil [19] presented the C-K theory according to which designing is not simply a problemsolving procedure; it is both a dynamic mapping process and a generation process for new objects. In the design process, the concepts were non-proven propositions that changed through the design process. Compared with other types of stimuli such as functional or form stimuli, IoT systems are dynamic systems, which may be the novelty of IoT systems stimuli. This helps in building an innovation design support tool based on IoT systems.

\section{Conclusion and Future Work}

In this research, we first present the model of using IoT systems as architecture design stimuli, then we used extended protocol analysis method to examine how participants use IoT systems as design stimuli. We found that participants prefer to use 'input part' as design stimuli, by analyzing the design results of participants, it is clear that both 'input part' and 'output part' in IoT systems have the promoter action to creativity, by analyzing the design process of participants, we found 'process part' in IoT systems enhance the extension of idea space.

Overall, this research indicates that using IoT systems as design stimuli is effective, we are motivated to establish the foundation for an innovation design support tool based on IoT systems. Future work includes examining the application of IoT systems to concept generation in more details.

\section{References}

[1] Karimi, K., \& Atkinson, G. (2013). What the Internet of Things (IoT) needs to become a reality. White Paper, FreeScale and ARM, 1-16.

[2] Cross, N. (2001). Strategic knowledge exercised by outstanding Designers. Strategic knowledge and concept formation III, 17-30.

[3] Chiu, I., \& Shu, L. H. (2007). Using language as related stimuli for concept generation. AI EDAM: Artificial Intelligence for Engineering Design, Analysis, and Manufacturing, 21 (02), 103-121.

[4] Benami, O., \& Jin, Y. (2002). Creative stimulation in conceptual design. In ASME 2002 international design engineering technical conferences and computers and information in engineering conference (pp. 251-263). American Society of Mechanical Engineers.

[5] Rothenberg, A. (1979). The emerging goddess: The creative process in art, science, and other fields.

[6] Lubart, T (1994) Creativity in R J Stenberg (ed) Thinking and problem solving, Academic Press, USA pp 289-332.

[7] Nagai, Y., Taura, T., \& Mukai, F. (2009). Concept blending and dissimilarity: factors for creative concept generation process. Design Studies, 30 (6), 648-675. 
[8] Taura, T., Nagai, Y., \& Tanaka, S. (2005). Design space blending-A key for creative design. In ICED 05: 15th International Conference on Engineering Design: Engineering Design and the Global Economy (p. 1481). Engineers Australia.

[9] Hampton, J. A. (1997). Emergent attributes in combined concepts. Creative thought: An investigation of conceptual structures and processes, 83-110.

[10] Costello, F. J., \& Keane, M. T. (2000). Efficient creativity: Constraint-guided conceptual combination. Cognitive Science, 24 (2), 299-349.

[11] Nagai, Y., \& Taura, T. (2006). FORMALDESCRIPTION OF CONCEPT-SYNTHESIZING PROCESS FOR CREATIVE DESIGN. In Design computing and cognition'06 (pp. 443460). Springer, Dordrecht.

[12] Taura, T., \& Nagai, Y. (2013). A systematized theory of creative concept generation in design: first-order and highorder concept generation. Research in Engineering Design, 24 (2), 185-199.
[13] Lawson, B. (1997). How Designers Think, ed.

[14] Ericsson, K. A., \& Simon, H. A. (1984). Protocol analysis: Verbal reports as data. the MIT Press.

[15] Finke, R. A., Ward, T. B., \& Smith, S. M. (1992). Creative cognition: Theory, research, and applications.

[16] Miller, G. A. (1995). WordNet: a lexical database for English. Communications of the ACM, 38 (11), 39-41.

[17] Varelas, G., Voutsakis, E., Raftopoulou, P., Petrakis, E. G., \& Milios, E. E. (2005, November). Semantic similarity methods in wordNet and their application to information retrieval on the web. In Proceedings of the 7th annual ACM international workshop on Web information and data management (pp. 1016). ACM.

[18] Howard, T. J., Dekoninck, E. A., \& Culley, S. J. (2010). The use of creative stimuli at early stages of industrial product innovation. Research in Engineering design, 21 (4), 263-274.

[19] Hatchuel, A., \& Weil, B. (2009). CK design theory: an advanced formulation. Research in engineering design, 19 (4), 181. 